\title{
RETRACTED ARTICLE: Albinism and mosaicism in Apocynum venetum associated with viral infections in China
}

\author{
Yanru Lan ${ }^{1,2,3} \cdot$ Zhichen Yan $^{1,2,3} \cdot$ Yane Guo ${ }^{1,2,3} \cdot$ Tingyu Duan $^{1,2,3}$ (1) Chunjie Li ${ }^{1,2,3} \cdot$ Peng Gao ${ }^{1,2,3}$. \\ Michael J. Christensen ${ }^{1,2,3}$
}

Received: 5 April 2018 / Accepted: 18 September 2018 / Published online: 28 September 2018

(c) Springer-Verlag GmbH Austria, part of Springer Nature 2018

The authors have retracted this article [1] due to flaws in the methodology which led to inaccurate results. Artefacts and environmental contamination were not experimentally excluded as the reasons for the positive detection of herpesviruses and bunyaviruses. As a consequence, false positive results were obtained so that misleading and unsupported conclusions were drawn. All authors agree to this retraction.

[1] Lan, Y., Yan, Z., Guo, Y. et al. Arch Virol (2018).

https://doi.org/10.1007/s00705-018-4059-4

Electronic supplementary material The online version of this article (https://doi.org/10.1007/s00705-018-4059-4) contains supplementary material, which is available to authorized users.

Tingyu Duan

duanty@1zu.edu.cn

1 State Key Laboratory of Grassland Agro-Ecosystems, Lanzhou University, Lanzhou, China

2 Agriculture Key Laboratory of Grassland Livestock Industry Innovation, Ministry of Agriculture and Rural Affairs, Lanzhou, China

3 College of Pastoral Agriculture Science and Technology, Lanzhou University, Lanzhou, China 